\title{
Die Medizin ist keine Wissenschaft
}

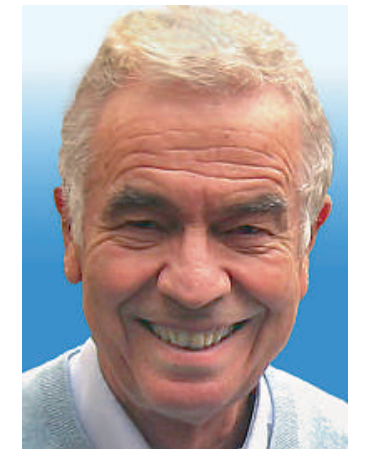

Hans Stalder
Im Studium der Medizin unterscheiden wir in der Regel zwischen Grundlagenwissenschaften und klinischen Wissenschaften. Grundlagenwissenschaften sollen das Fundament sein, auf das die Klinik aufbaut. Nach der angelsächsischen Unterscheidung der Wissenschaften in Naturwissenschaft, Sozialwissenschaft und Geisteswissenschaft erscheint es evident, dass die Grundlagenwissenschaften zu den Naturwissenschaften zu zählen sind. Das naturwissenschaftliche Verständnis vom Funktionieren des Körpers und - in jüngerer Vergangenheit - des Gehirns, bildet die Basis für die spektakulären medizinischen Fortschritte der letzten beiden Jahrhunderte. Die Bedeutung der Naturwissenschaften zeigt sich auch in der steigenden Anzahl von Dozenten der Biologie mit einem Abschluss als PhD an unseren Fakultäten, die - obwohl sie medizinische Grundlagenwissenschaften unterrichten - die Medizin häufig nur als Patient kennen. Und in Lausanne heisst die medizinische Fakultät seit einigen Jahren Faculté de biologie et de médecine.

Und doch - hat der Student nicht ein bisschen recht, wenn er seinen Bachelor of Medicine (vormals 3. Propädeutikum) in der Tasche hat und meint, «jetzt kann ich alles vergessen und endlich wirkliche Medizin studieren»? Natürlich soll er nicht alles vergessen, denn seine künftige Tätigkeit basiert auf den wohlfundierten Grundlagen der Biologie. Aber er hat doch ein bisschen recht, denn die «wirkliche Medizin», die er eines Tages praktizieren möchte, stützt sich nicht allein auf die Biologie. Anhand einiger Beispiele lässt sich aufzeigen, dass die Medizin ein breiteres Fundament braucht als nur die Naturwissenschaften.

Beim Alkoholiker, der das Trinken nicht aufgeben will, kommt bei der Ausarbeitung des Motivationsgesprächs die Pädagogik ins Spiel. Etwas mehr Pädagogik wäre im Übrigen nicht nur für Professoren nützlich, sondern auch für praktizierende Ärzte, die immer mehr Lehrfunktionen übernehmen. Und der Patient, der mir wegen seines suizidalen Verhaltens Angst einjagt? Die Instrumente, die helfen, ihn zu verstehen und meine Ängste zu überwinden, stammen aus der Psychologie. Wenn ich meinen Patienten Ratschläge zur Vorbeugung geben will, sollte ich mit Hilfe der Sozialwissenschaft meine Anamnese vervollständigen, denn die umweltspezifischen und sozioökonomischen Bedingungen sind mindestens so wichtig wie die biologischen Risikofaktoren. Um mit einem kritischen Geist Artikel über die Vorteile dieses oder jenes Medikaments im Vergleich zu anderen oder zu Plazebo lesen zu können, bedarf es epide- miologischer Kenntnisse. Ein Fünftel der Schweizer Bevölkerung sind Ausländer mit einem anderen kulturellen Hintergrund. Um sie verstehen zu können, benötige ich einige Begriffe aus der medizinischen Anthropologie. Kann ich einem Kranken mit «funktionellen» Störungen ein Plazebo verabreichen? Das ist eine ethische Frage. Daher benötige ich auch ge-

\section{«Doch ihre Dozenten sind eine kleine, zu vernachlässigende Minderheit.»}

wisse Grundlagen in medizinischer Ethik. Und schliesslich helfen die Medical Humanities dort, wo es die anderen Wissenschaften nicht können: die Philosophie, um anders zu denken, die Geschichte, um anders zu sehen, die Kunst, um sich anders auszudrücken; damit der Arzt wird, wie ihn sich die Bevölkerung vorstellt: ein Humanist.

Beinahe hätte ich die Wirtschaftswissenschaften vergessen, wenngleich ihre Bedeutung vielen erst bei der Praxiseröffnung deutlich wird, wenn sie den Herausforderungen von TARMED und Managed care gegenüberstehen ...

Erfreulicherweise können wir feststellen, dass Schritt für Schritt auch Sozialwissenschaften, Psychologie, medizinische Ethik, Anthropologie und Medical Humanities Einzug ins Medizinstudium halten. Doch ihre Dozenten sind eine kleine, zu vernachlässigende Minderheit und eine «Fakultät der Soziologie und der Medizin» oder eine «Fakultät der Geisteswissenschaften und der Medizin» wird es in allernächster Zukunft noch nicht geben. Heute, wo Universitäten um ausserbudgetäre Ressourcen kämpfen, wäre dies auch wenig erfolgreich, denn diese Bereiche sind einfach nicht die, where the money is ... Dann vielleicht gemäss dem Beispiel der Genfer Regierung, in der Wirtschaft und Gesundheit im selben Departement untergebracht sind, eine «Fakultät der Wirtschaft und der Medizin»?

Bleiben wir dabei: Die Medizin ist keine Wissenschaft. Medizin gründet vielmehr auf vielfachen wissenschaftlichen Fundamenten und Grundlagen. Und deren Vermittlung bleibt eine Herausforderung für unsere Fakultäten.

Hans Stalder* 